\title{
XLVI ISCEV International Symposium
}

\author{
Morgantown, West Virginia. 10-15 July 2008
}

(C) Springer-Verlag 2008

Thursday, July 10, 2008

7:30 a.m.-5:30 p.m.

Registration - Foyer A B C

12:00 p.m.

Board Meeting with Lunch - Puskar Boardroom 5:00-7:00 p.m.

Clinical Cases Session - Salon A, B, C, D

7:30 p.m.

Reception - Foyer FGH

Friday, July 11, 2008

7:00-8:30 a.m.

ISCEV Approved Protocols Committee-Eberhart Zrenner, Chairing Session

Puskar Boardroom

7:30 a.m. $-5: 30$ p.m.

Registration - Foyer A B C

8:30 a.m.-10:00 a.m.

Opening-Welcome by Organizers, J. Vernon Odom and Monique Leys;

Welcome by Judie Charlton, MD, Chair of Ophthalmology; Opening by Michael Bach, President of ISCEV

Salon A, B, C, D
8:45 a.m.

ORAL SESSION 1. Correlation of Cortical Structure and Function

Chairs: Michael Bach and J. Vernon Odom

8:45 a.m.

INVITED LECTURE 1. Studies of Visual Social Cognition

Aina Puce

9:15 a.m. OS1-1 Multi-Harmonic Objective

Response Detection in Steady-State Evoked

Potentials Using Permutation Techniques

Sven P. Heinrich and Michael Bach

9:30 a.m. OS1-2 Attentional Shifts have Little

Effect on the Waveform of the Chromatic Onset VEP

Michael A. Crognale and Jennifer Highsmith 9:45 a.m. OS1-3 Color and Orientation Processing in Macaque Area V2: Differential Response Characteristics Defined by Single Unit Electrophysiology and Optical Imaging Benjamin M. Ramsden and Clinton G. Cooper 10:00 a.m. - Break 
10:30 a.m.-12:00 p.m.

ORAL SESSION 2. Insight into Retinal

Function-Animal Models

Chairs: Laura Frishman and Pierre Lachapelle

10:30 a.m. OS2-1 Transgenic Rabbit Model of

Retinal Degeneration

Mineo Kondo, Taka Sakai, Keijchi Komeima, Yukihide

Kurimoto, Yuji Nishizawa, Jiro Usukura, Takashi

Fujikado, Yauso Tana, Hirko Terasaki

10:45 a.m. OS2-2 Vitamin A-Dependent Shift of

Photoreceptor Sensitivity in the ERG of RPE65

Mouse Mutants

Naoyuki Tanimoto, Marijane Samardzija, Johannes von Lintig, Vitus Oberhauser, Markus Thiersch,

Charlotte E. Reme, T. Michael Redmond, Christian

Grimm, Andreas Wenzel, Mathias Seeliger.

11:00 a.m. OS2-3 Compromised Cone System

Functions in MDX3CV Mice, a Model for

Duchenne Muscular Dystrophy

Bo Lei, Keqing Zhang, Yongping Yue, Dongsheng

Duan

11:15 a.m. OS2-4 Retinal Pathway Origins of the Pattern ERG (PERG)

Xunda Luo, Gen Miura, Minhua Wang, Laura J.

Frishman

11:30 a.m. OS2-5 Photoreceptor Contributions to

the full field Photopic Flicker Electroretinogram of the Rat

Suresh Viswanathan, Christina Gant

11:45 a.m. OS2-6 Understanding the Origins of the Mouse DC-Electroretinogram

Neal S. Peachey

12:00 p.m. - Lunch

1:00 p.m.-2:30 p.m.

POSTER SESSION A. Pediatrics-ERG and VEP Methods

Chairs: Anne Fulton and Thomas Meigen

PSA-1 The Red Electroretinogram Response, and its Relationship with Age, Gender, Body Mass Index, and Axial Length, in Normal Chinese Eyes

Winnie Wai Ying Lau, Albert Chak Ming Wong,

Edwin Chan, Clement Wai Nang Chan
PSA-2 Early Diagnosis of Organic Amblyopia in Children Using Electroretinography

M.S. Mridehghan, S.M. Shushtarian, M. Mirdenghan

PSA-3 Recording of Electroretinogram and Visual Evoked Potential Under Anesthesia

Mohammad Mehdi Shushtarian, Seyed Mohammad Masoud Shushtarian, Soheil Feizi, Tahereh Parsa, Alireza Khodyani

PSA-4 $30 \mathrm{~Hz}$ and Mixed Response Electroretinogram done during Anaesthesia as Predictors of Visual Outcome in Children Affected by Various Eye Diseases: 10-Year Survey

Giulio Ruberto, Rosanna Guagliano, Donatella Barilla, Mauro Antonimi, Sara Pezzotta, Sabrina Signorini, Elisa Fazzi, Raffaella Angeli, Carmine Tinelli, Paolo Emilio Bianchi

PSA-5 Comparison of Electroretinographical Patterns in Normal Children and Adult Population

H. Hassani, S.M. Shushtarian

PSA-6 Chromatic VEP in the First Year of LifeA Longitudinal Study

Manca Tekavcic Pompe, Branka Stirn Kranjc, Jelka Brecelj

PSA-7 Neonatal Flash VEPs: Effect of Exposure to Methadone in Utero

Ruth Hamilton, Laura McGlone, Helen Mactier, Michael Bradnam, Richard Boulton, William Borland, Mary Hepburn, Daphne McCulloch

PSA-8 Electrophysiological Impairment is Associated with Biochemical and Histological Modifications in the Retina of Chronic Alcoholic Rats

M. Sancho-Tello, M. Muriach, J. Barcia,

F. Bosch-Morell, J.M. Genoves, S. Johnsen-Sorano,

B. Romero, I. Almansa, M. Diaz-Liopis,

S. Garcia-Delpech, J. Roma, C. Vilela, F.J. Romero

PSA-9 Photopic On- and Off-Responses in Normal

Korean Subjects

Soo Young Lee, Su Eun Park, Young-Hoon Ohn

PSA-10 Changes of Retinal Adaptation in Myopic Eye

Wing-cheung Ho, Yiu-fai Ng, Patrick Ho-wai Chu, Ying-ying Fong, Kwun-sum Yip, Henry Ho-lung Chan

PSA-11 Would Increasing Flash Intensity be Sufficient to Yield Similar ERG Amplitudes in the Non-dilated Eye Compared to the Dilated Eye? 
Marc Hebert, Marie-Pier Lavoie, Marie-Claude

Charron, Alexandre Sasseville, Anne-Marie Gagne

PSA-12 Effect of Docosahexaenoic Acid Supplementation on Retinal Function in ELOVL4 Mice

S.C. Mema, S. Kuny, F. Gaillard, K. Zhang,

I.M. MacDonald, M.T. Clandinin, Y. Sauve

PSA-13 Electrophysiological Testing-Our Early Experience

Irene Sophia Joseph, Anupama Rao, B.S. Chidamber

PSA-14 Intra-Sessional and Inter-Sessional

Variability of Multifocal Electroretinogram

Uzeyir Erdem, Fatih Cakir Gundogan, Gungor

Sobaci, Mehmet Zeki Bayraktar

PSA-15 Establishment of Normal Ranges for the ISCEV Standard Visual Evoked Potential

Enid Chelva, Doug Candy, John De Roach,

Terri McLaren, Maribel Nosce, Roger Price

PSA-16 Steady State Pattern VEPS: Onset-Offset

Checks are More Effective than Pattern Rever-

sal for Frequency Domain Detection

Michelle A. McIntosh, Elena Prokofyeva, Richard

G. Boulton, Daphne L. McCoulloch

PSA-17 Stroboscopic and Square Wave Flicker

VEPS for Visual Pathway Assessment

Elena Prokofyeva, Michelle McIntosh, Richard

G. Bourlton, Daphne L. McCulloch

PSA-18 Effects of Anisometropia on Binocular Integration in Normal Observers

L. Lefebvre, D. Saint-Amour

PSA-19 A Comparison of Visual Acuity Estimates from Visual Evoked Cortical Potentials, FRACT and ETDRS

A. Kurtenbach, H. Langrova, A. Messias, E. Zrenner, H. Jägle

PSA-20 VEP Asymmetry vs. Chiasm Coefficient in Children with MRI confirmed Achiasmia

Jelka Brecelj, Maja Šuštar, Branka Stirn-Kranjc, Nuška Pečarič-Meglič, Miha Škrbec

3:00 p.m.-5:00 p.m.

ISCEV Olympics-Ruby McQuain Park

5:30 p.m.

Walking dinner with ISCEV Band-Foyer FGH/ Patio

Participants may choose an Evening Activity:

Musical or "Film in the Park"

7:30 p.m.

Buses Leave for West Side Story
8:00 p.m.

Westside Story

9:00 p.m. "Film in the Park"

Movie "Unaccompanied Minors"

Saturday, July 12, 2008

7:30 a.m.-5:30 p.m.

Registration - Foyer A B C

7:00-8:30 a.m.

Springer - Documenta Ophthalmologica - Editorial

Board Meeting-Puskar Boardroom

8:30-10:00 a.m.

ORAL SESSION 3. Low Vision and Retinal

Function

Chairs: William Seiple and Sandeep Grover

8:30 a.m.

INVITED LECTURE 2. REHABILITATION IN VISUALLY IMPAIRED CHILDREN: “Coming to Better Terms....with"

Frans CC Riemslag

9:00 a.m. OS3-1 To Assess the Functional Activity of the Outer Retinal Layers at the Pseudofovea in Eccentric Fixation

Ajoy Vincent, Rohit Shetty, Vasudha Naresh,

M. Bhanumathi, Bhujang Shetty

9:15 a.m. OS3-2 Micro-Current Neuromodulation in the Management of Dry Age-Related Macular Degeneration

Sundaram Natarajan, Mahesh Uparkar, Anjali

Khulsange, Darshana Gadgil

9:30 a.m. OS3-3 Delayed Implicit Time of Pattern

Visual Evoked Potentials in Patients with

Occult Macular Dystrophy

Kazhua Ogata, Shuichi Yamamoto

9:45 a.m. A Causal Role for the Rods in

Retinopathy of Prematurity

James D. Akula, Ronald M. Hansen, Tara L. Favazza,

Tanya C. Vyhovsky, Ilan Y. Benador, Julie A.

Mocko, Anne B. Fulton

10:00 a.m. Break - Foyer FGH 
10:30 a.m.-12:00 p.m.

ORAL SESSION 4. Evaluation of Toxicity and Visual Function

Chairs: Daphne McCulloch and Mineo Kondo

10:30 a.m. OS4-1 ERGS During Examination-

Under-Anesthesia: Responses of Normal Fellow Eyes

Scott Brodie, Dawn Desiderio, David H. Abramson

10:45 a.m. OS4-2 Postnatal Exposure to Bright

Light Stimulates Retinal Plasticity

P. Lachapelle, A. Polosa, E. Zimak, W. Liu,

L. Robbe, S. Joly, S.G. Rosolen, S. Chemtob

11:00 a.m. OS4-3 Long-Term Methamphetamine

Abuse Influences Visual Evoked Potentials

Jan Kremláček, Miroslav Kuba, Ladislav Hosák, Jan

Libiger, Jiří Č́ížek

11:15 a.m. OS4-4 ERG Effects of Chronic (Daily)

Sildenafil or Tadalafil Usage

Michael F. Marmor, Christopher Zoumalan, Roham

T. Zamanian, Ramona L. Doyle, William

H. Cordell

11:30 a.m. OS4-5 Is it Possible to use the Mf ERG to Follow-Up Hydroxychloroquine Therapy in Elderly Patients?

I. Ingster-Moati, E. Bui Quoc, C. Orssaud,

D. Schmitt, Ph. Le Neindre, J.L. Dufier, O. Roche

11:45 a.m. OS4-6 Using Multifocal ERG Ring

Rations to Establish Optimal Dosage Criteria

for Hydroxychloroquine Retinal Toxicity

Jonathan S. Lyons, Matthew L. Severns

12:00 p.m. LUNCH - Salon EFGH

1:00-2:30 p.m.

ORAL SESSION 5. Optic Nerve and Glaucoma

Chairs: Mitchell Brigell and Graham Holder

1:00 p.m. OS5-1 Visual Electrodiagnosis and Visual

Outcome in Optic Nerve Hypoplasia (ONH)

Daphne L McCulloch, Pamela Garcia-Filion,

Cassandra Fink, Caroline A. Chaplin, Mark

S. Borchert

1:15 p.m. OS5-2 The Optic Nerve Head Component of the Porcine Multifocal Electroretinogram Rita Buffa, Aphrodite Dracopoulos, Shelley Boyd
1:30 p.m. OS5-3 Decrease in Sweep VEP Acuity and Contrast Sensitivity in Ocular Hypertensive Rabbits - Induced by Application of Carbopol

K-M Zhang, L. Ruiz, A. Vilupuru, B. Feldmann, W. Orilla, T. Lin, Y. Li, C. Spada, M. Escobar, C. Ghosn, J. Burke

1:45 p.m. OS5-4 Long-Term Study of Multifocal Electroretinographic Changes In Experimental Glaucoma in Macaques

T. Michael Nork, Charlene B. Y. Kim, Gregg

A. Heatley, Paul L. Kaufman, Mark J. Lucarelli, Leonard A. Levin, James N. Ver Hoeve

2:00 p.m. OS5-5 Multifocal-OPs of a two Global Flash Stimulus in POAG and NTG

Anja M. Palmowski-Wolfe, Margarita G. Todorova, Selim Orguel, Josel Flammer

2:15 p.m. OS5-6 Pattern ERG in Glaucoma

Suspects-New Findings from a Longitudinal Study

Michael Bach, Thomas Jehle

2:30 p.m. BREAK

3:00 p.m.-4:30 p.m.

ORAL SESSION 6. Frontiers in

Electrophysiological Testing

Chairs: Carol Westall \& David Keating

3:00 p.m. OS6-1 The Single and Multifocal

M-Sequence Technique and Their Uses in Visual Electrophysiology

Erich E. Sutter

3:15 p.m. OS6-2 Mydriasis does not Impair Multifocal VEP Recordings

Thomas Meigen, Anneliese Friedrich

3:30 p.m. OS6-3 Accommodation Limits Induced Optical Defocus in Defocus Experiments Herbert Jägle, Thomas Brauns

3:45 p.m. OS6-4 Intricacies of Measuring the mfERG's Luminance Uniformity in Direct and Maxwellian Viewing

Fabrice Moret, Wolf A. Lagrèze, Michael Bach 4:00 p.m. OS6-5 Light Adaptation and Dark Adaptation of the Human Visual System

Rasa Ruseckaite, Allison M. Cameron, Liang Miao, Trevor D. Lamb 
4:15 p.m. OS6-6 Precisely Specifying the Bandwidth of Electrodiagnostic Recordings

A.C. Fisher, S.P. Lake, R.P. Hagan, M.C. Brown

\section{4:30-6:30 p.m.}

POSTER SESSION B. Retinal Diseases ERG Methods

Chairs: Monique Leys and Neal Peachey

PSB 1 Study of Cone Dysfunction in Eyes with Macular Edema

Weiju Wu, Feng Wen, Shizhou Huang, Guangwei Luo, Dezheng Wu

PSB 2 Multifocal Electroretinographic and

Electroretinographic Change of Affected and

Fellow Eye with Central Retinal Vein Occlusion (CRVO)

Manami Kuze, Hisashi Matsubara, Yukitaka Uji

PSB 3 Foveal Thinning in Cases with Occult

Macular Dystrophy

Azza Shehab, Mai Sharawy

PSB 4 Local and Multifocal Electroretinography and Macula Imaging in Stargardt's Disease/

Fundus Flavimaculatus

I. Zolnikova, E.V. Rogatina, I.Z. Karlova

PSB 5 Changes in the Myopic Retina Using

Multifocal Electroretinograms, Optical Coherence Tomography and Peripheral Resolution Acuity

Clive J. Wolsley, Kathryn J. Saunders, Giuliana Silvestri, Roger S. Anderson

PSB 6 Spontaneous Recovery of the Photoreceptor Inner/Outer Segment Line Defect in Eyes with Multiple Evanescent White Dot Syndrome

Atsuhiro Tanikawa, Eri Nakayasu, Ryo Asano, Masayuki Horiguchi

PSB 7 Bilateral Choroidal Neovascular Membrane as a Presenting Feature of Bests Disease

Rohit Shett, Ajoy Vincent, Rajani R Battu, Bhujang Shetty

PSB 8 Dark Adaptometry Following Implantation of Blue Blocker and Aspheric Intraocular Lenses

Rohit Shetty, Mathew Kurian, Ajoy Vincent, Bhujang Shetty

PSB 9 Syndromic Choroideremia-A Possible Approach to Sublocalization of Phenes Associated with Martin-Probst Deafness Mental Retardation Syndrome

C.M. Poloschek, B. Kloeckener-Gruissem, L.L. Hansen, M. Bach, W. Berger

PSB 10 The Importance of Clinical Examination of Patients who come for Electrofunctional Test Alma Patrizia Tormene, Morena Masetto, Chiara Riva PSB 11 Skin Electrodes vs. Corneal Electrodes (Burian-Allen) for ISCEV Standard Full field ERG-Determining a Method to Obtain Repeatable Results

Mary Flynn Roberts, Elizabeth Warren, Angela VanDyke

PSB 12 Electrical Impedance Measurement on the Sclera/Cornea

Malcolm Brown, Laura Milner, Anthony Fisher, Richard Hagan

PSB 13 the Insulating Effect of Silicon Oil in Vitrectomized Human Eyes

Ajoy Vincent, Rohit Shetty, Naresh Kumar Yadav, Priya B Vijayan, Sri Bhargav Natesh, Bhujang Shetty PSB 14 A Promising S-cone Isolating Protocol Josefin Nilsson, Thomas Wright, Carol Westall PSB 15 The Assessment of the Single-Flash ERG A-Wave Slope by Determination of Derivatives in Different Time Points

Sergey Rezvykh, Irina Tsapenko, Igor Spiridonov, Marina Zueva

PSB 16 Software for the Evaluation of Electroretinograms

Andre Messias, Herbert Jagle, Florian Gekeler and Eberhart Zrenner

PSB 17 Assessment of Human Dark Adaption Kinetic Using the Electoretinogram B-Wave Amplitude and Dark Adaptometry

Susanne Kramer, Andre Messias, Eberhart Zrenner and Herbert Jagle

PSB 18 The Photopic Negative Response of the Red on Blue Electroretinogram Using LED Built-in Contact Lens Electrodes on Open Angle Glaucoma.

Kaoru Fujinami, Rishu Inoue, Toshine Maeda, Toyoka Inoue, Yoichi Inoue, Kazushige Tsunoda, Yozo Miyake

PSB 19 Investigation of Photopic Negative Response in Glaucoma by Focal Electroretinograms

Hajime Nakamura, Masanori Hangai, Fumitaka Hirose, Tomonari Ojima, Nagahisa Yoshimura 
PSB 20 Microcurrent Neuromodulation in

Retinitis Pigmentosa: Report of a Pilot Study

Subhadra Jalali, Raja Narayanan

PSB 21 The Leber Congenital Amaurosis Protein, AIPL1, Plays a Direct Role and is Indispensable for Cone Photoreceptor Cells

Lindsay Michalovicz, Saravanan Kolandaivelu, Visvanathan Ramamurthy

PSB 22 Treatments of Neovascular Age-Related Macular Degeneration: A Quantitative Review

J. Vernon Odom, PhD, Ahmed El-Sherbeeny, $\mathrm{PhD}$ and Nabil Jabbour, MD

FREE EVENING: Dinner on Your Own

Sunday, July 13, 2008

8:00 a.m. Leave for Fallingwater

9:30 a.m. Arrive at Fallingwater/Break

10:00 a.m. Introduction-Fallingwater Barn

10:15 a.m.

ORAL SESSION 7. Retinal Structure and Function

Chairs: Jonathan Lyons and Masayuki Horiguchi

10:15 a.m.

INVITED LECTURE 3. Low Vision Care and Electrophysiology: From a Clinical Point of View

Kenji Yanashima

11:00 a.m. OS7-1 Objective Assessment of Retinal Functions of Subjects with Advanced Retinal Degeneration in Clinical Trials

R. Wilke, M. Bach, A. Schatz, T. Strasser, H. Benav, A. Messias

11:15 a.m. OS7-2 Clinical and Electrophysiologic Characteristics of a Large Kindred with $\mathrm{X}$ Linked Retinitis Pigmentosa Associated with the RPGR Locus

Byron L. Lam, Audina Berrocal, Tania Arguello, William Feuer, Mu Liu, Ditte Hess, Michelle Caputo, Maria Berrocal, Alexandra Weisman, Edwin M. Stone

11:30 a.m. OS7-3 Meeting the Challenge of Reliable Electrophysiological Retinal Function Testing in Multi-Centre Trials: State of
Development of the "ISCEV Protocols for Clinical Trials"

Eberhart Zrenner

11:45 a.m. Lunch

12:45 p.m. Departure for Tours

1::00 p.m. Fallingwater/Kentuck Knob

6:30 p.m. Dinner (Barbeque- Roast)

Music by Stewed Mulligan and Square Dance called by Taylor Runner

9:30 p.m. Leave for Morgantown

11:00 p.m. Arrive at Morgantown

Monday, July 14, 2008

8:30 a.m.-5:30 p.m.

Registration - Foyer ABC

8:30 a.m.-10:00 a.m.

ORAL SESSION 8. Imaging and Visual Electrophysiology

Chairs: Marko Hawlina and Colin Barber

8:30 a.m.

INVITED LECTURE 4. Inner Retinal Sequelae of Outer Retinal Disease: Analysis of ERG Components in the RCS Rat

Paul A. Sieving (with Ronald A. Bush, Dorit Raz, Shigeki Machida)

9:00 a.m. OS8-1 Simultaneous Recording of NearInfrared Spectroscopy and Visual Evoked Potentials in Normal Human Subjects

Kazuhiko Tanabe, Atsushi Mizota, Minoru Tanaka, Ichiro Shimoyama

9:15 a.m. OS8-2 Association between Functional Markers and Structural Retinal Changes in Adolescents with Type I Diabetes

Ekta Lakhani, Thomas Wright, Giuseppe Mirabella, Peter Glazer, Carol Westall

9:30 a.m. OS8-3 Sensitivity and Specificity of PERG in Relation to Stimulus Field Size and OCT in Patients with Stargardt's Disease

Eva Lenassi, Darko Perovšek, Martina Jarc-Vidmar, Petra Popovič, Marko Hawlina 
9:45 a.m. OS8-4 Multimodal Imaging: A New Tool to Investigate the Relationship between Macular Structure and Function in Retinal Disease S.M. Walker, S. Parks, D. Keating 10:00 a.m. Break

10:30 a.m.-12:15 p.m.

\section{ORAL SESSION 9. Hereditary Retinal Diseases}

Chairs: Alma Patrizia Tormene and Enid Chelva 10:30 a.m. OS9-1 A Novel Approach Analyzing

Multifocal ERGS: Spatiotemporal Partial Least Squares (ST-PLS)

Thomas Wright, Filomeno Cortese, Carol Westall 10:45 a.m. OS9-2 On the Differentiation between Fundus Albipunctatus and Retinitis Punctata Albescens

G.I. Holder, E. Sohn, L. Zheng, V.M. McBain,, A.G. Robson, A.R. Webster,

11:00 a.m. OS9-3 Progression of Retinal Dystrophy in Mucolipidosis IV-An Electroretinographic Study

Rafael C. Caruso, Raphael Schiffmann, Janine A. Smith, Ehud Goldin

11:15 a.m. OS9-4 Negative ERG in a Patient with Subacute Sclerosing Panencehalitis

Hiroko Yamazaki, Chai Yuzhu, Yutaka Nonoda, Asako Arai, Eiji Nakagawa, Makiko Kaga

11:30 a.m. OS9-5 CSNB Caused by a Novel Nonsense Mutation in CABP4 is Characterized by Severe Cone Dysfunction, but Normal Scotopic Vision and Normal Primary Rod Pathway

Maria van Genderen, Karin Littink, Frans Riemslag, Frans Cremers, Ingeborgh van den born

11:45 a.m. OS9-6 The Sensitivity and Specificity of ISCEV Standard ERGS and EOGs when Discriminating Normal, Retinitis Pigmentosa, and Mixed Disease

Vaegan and Stephanie Yeo

12:00 p.m. OS9-7 Principal Component Analysis of Multifocal Electroretinogram in Retinitis Pigmentosa

Aniruddha Maiti, Mahesh Uparkar,

Sundaram Natarajan

12:15 p.m. Lunch - Salon EFGH
1:00 p.m.-3:00 p.m.

POSTER SESSION C. Electrophysiological

Testing: EOG, MFERG, VEP Methods

Chairs: Subhatra Jalali and Scott Brodie

PSC-1 Hypotension and Electro-oculogram

Yuzo Myake, Atsuhiro Tanikawa, Mitsuo Sugimoto, Masayuki Horiguchi

PSC-2 Bilateral Maculopathy with Subretinal

Neovascularization in a Patient with

Charcot-Marie Tooth Disease

Richard MacMaster Francis, Jr., Lionel Chisholm, Monique Leys

PSC-3 Retinal Function in Diabetic Retinopathy with Macular Edema

Mariam Arakelian, Natalia Mansurina, Yulia Bardeeva, Angelika Shamshinova

PSC-4 Characteristics of Global Flash Multifocal Electroretinogram (mfERG) under Different Combinations of Global and Focal Flash Luminance

Jenny CY Lung, Patrick HW Chu, YF Ng, Henry HL Chan

PSC-5 The Techniques and the System for Multifocal Electroretinography

Aram Arakelyn

PSC-6 Electrophysiological and Morphological Changes after Intravitreal Bevacizumab Injection in Patients with Macular Edema and Choroidal Neovascularization

Young-Hoon Ohn, Soo Young Lee, Hyun Joon Lee PSC-7 Multifocal Electroretinogram Progression in Patients with RP

Karen Holopigian, Jenny M. Gallardo, William Seiple, William H. Swanson, Ronald E. Carr

PSC-8 Foveal Dystrophy or Dysfunction: A Clinical Entity

N. Khan, J.R. Heckenlively

PSC-9 Correlation between Vertical Kinetic

Visual Acuity and Visual Evoked Potential in Patients with Unilateral Optic Neuritis

Yujin Mochizuki, Atsushi Mizota, Minoru Tanaka, Yoshimitsu Kohmura, Hiroshi Yoshigi

PSC-10 Blue Fixation Stimulus to Record Pattern VEP in Optic Nerve Disease

Rohit Shetty, Ajoy Vincent, Bhujang Shetty

PSC-11 Abnormal Pattern Visual Evoked Potential in Occult Macular Dystrophy-Is it the Nerve? 
Ranjana Mathur, Doric Wong, Kuang Wen

PSC-12 Recording of Visual Evoked Potential in Photophobia Patients

Maryam Mirdehghan, S.M. Shushtarian,

M.S. Mirdehghan

PSC-13 VEPS in Response to Cone Isolating Stimuli

in College Students with Reading Discomfort

William H. Ridder, III, Christopher Chase, Chinatsu

Tosha, Anna Tong, Dani Smith, Eric Borsting

PSC-14 Effect of Whole Body Vibration on Visual

System Using Visual Evoked Potentials

Aryandokht Fotros, Seyed Mohammad Mehdi

Shushtarian

PSC-15 Retinal (Macular) Diseases are

Distinguished Easier from Optic Neuropathies

if Electrophysiology is Complemented by

Optical Coherence Tomography (OCT)

Hannes Wildberger, Daniel Barthelmes, Oliver

Bergamin

PSC-16 Effects of Nicotine on the Adult

Electroretinogram

S.B. Varghese, E.E. Hartmann, J.C. Reid,

K.T. Keyser

PSC-17 The Fast and Slow Oscillations of the EOG in Diabetes

Marilyn E. Schneck, Leslie Shupenko and

Anthony J. Adams

PSC-18 ERG Response Smearing into 'Unused'

M-Sequences at Fast Stimulation Rates

R.P. Hagan, A.C. Fisher, M.C. Brown

PSC-19 Transfer Functions for Rescaling

Electroretinograms Obtained with Two

Different Translid DTL Electrodes

Vaegan, Anthony C. Fisher and Tom Wright
PSC-20 Retinal Functional and Morphometric

Analysis of Intravitreal Triamcinolone Acetonid Injection in Diffuse Diabetic Macular Edema:

Optical Coherence Tomography and Multifocal Electroretinogram Study

Fatih C. Gundogan, Serdar Memisoglu,

Ali H. Durukan.

2:30-3:00 p.m.

Poster Session Continues with Break - Foyer FGH

3:00-3:45 p.m.

The EMIKO ADACHI LECTURE 2008

Adachi Award Introduction: Joseph Harrison

Maculas, Monkeys, Metabolism, Aging, and AMD

William W. Dawson

4:00-5:30 p.m. Membership Meeting

6:30-7:00 p.m. Reception

7:00-11:00 p.m.

Gala Dinner

8:00 p.m. Recognition of Local Organizers

Awards: Dodt Award; Adachi Award

Mitchell Brigell

ISCEV OLYMPICS: Colin Barber as MC

9:00 pm. Music and Dancing

Tuesday, July 15, 2008

9:30 a.m.-12:00 p.m. Tour of WVU Eye Institute 12:00-1:00 p.m. Lunch - John Jones Conference Center

Social Activities

ISCEV Golf Tournament 